\title{
Design And Characterization of an Electrically Powered Single Molecule on Gold
}

\author{
Rémy Pawlak, ${ }^{* \dagger}$ Tobias Meier, $^{\dagger}$ Nicolas Renaud, ${ }^{\ddagger}$ Marcin Kisiel, ${ }^{\dagger}$ Antoine \\ Hinaut, ${ }^{\dagger}$ Thilo Glatzel, ${ }^{\dagger}$ Delphine Sordes, ${ }^{\top}$ Corentin Durand, "We-Hyo Soe, \\ Alexis Baratoff, ${ }^{\dagger}$ Christian Joachim, ${ }^{\Uparrow}$ Catherine E. Housecroft, ${ }^{\S}$ Edwin C. \\ Constable,,$^{\S}$ and Ernst Meyer ${ }^{\dagger}$ \\ $\dagger$ Department of Physics, University of Basel, Klingelbergstr. 82, 4056 Basel, Switzerland \\ $\ddagger$ Delft University of Technology, Department of Chemical Engineering, van de Maasweg 9, \\ 2629 HZ Delft, The Netherlands \\ \CEMES-CNRS, NanoSciences Group 83 MANA Satellite, 29 rue Jeanne Marvig, BP \\ 94347, F-31055 Toulouse cedex 4, France. \\ $\S$ Department of Chemistry, University of Basel, Spitalstrasse 514056 Basel, Switzerland \\ E-mail: remy.pawlak@unibas.ch
}

\begin{abstract}
The surface diffusion of individual molecules is of paramount importance in selfassembly processes and catalytic processes. However, the fundamental understanding of molecule diffusion peculiarities considering conformations and adsorption sites remain poorly known at the atomic-scale. Here, we probe the 4'-(4-tolyl)-2,2':6',2"terpyridine adsorbed on the $\mathrm{Au}(111)$ herringbone structure combining scanning tunneling microscopy and atomic force microscopy. Molecules are controllably translated by electrons excitations over the reconstruction, except at elbows acting as pinning
\end{abstract}


centres. Experimental data supported by theoretical calculations show there the formation of coordination bonds between the molecule and Au atoms of the surface. Using force spectroscopy, we quantify local variation of the surface potential and the lateral force required to move the molecule. We found an elevation of the diffusion barrier at elbows of the reconstruction of $\sim 100 \mathrm{meV}$ compared to the rest of the surface.

Keywords: atomic force microscopy, single-molecule, scanning tunneling microscopy, force spectroscopy, molecular machine.

"Special problems occur when things get small [...] and might turn out to be advantages if you knew how to design for them." commented R. Feynman in 1983 while discussing the prospects of building-up "infinitesimal machinery". ${ }^{1}$ In spring 2017 , the first international "NanoCar Race" was organized by the CEMES laboratory ${ }^{2}$ in a unique low-temperature four-probe scanning tunneling microscope (STM). The competition aimed to propel speciallydesigned molecular vehicles along the $\mathrm{Au}(111)$ herringbone structure. The race took place at the nanometer scale during 36 hours between four participants "driving" in parallel their concept cars. In addressing this challenge, the competitors had to tackle the fundamental issues expressed by Feynman in his seminal speech: the proper design of such "machinery", its actuation and direction of motion at the atomic scale as well as the control of the friction forces arising at the surface.

With recent progress in scanning tunneling microscopy (STM) and atomic force microscopy (AFM) at low temperature, the investigation of organic compounds at surfaces has become possible from the single-molecule level up to their large-scale assemblies with remarkable precision. ${ }^{3}$ These techniques have also demonstrated their abilities to induce local displacements of single-atoms and molecules down to the atomic level ${ }^{4}$ through precise repositioning by mechanical interactions. ${ }^{5-7}$ This real-space approach further allowed insights into intramolecular mechanics, ${ }^{8,9}$ single-molecule tribology properties ${ }^{10-13}$ as well as the design and manipulation of elaborated molecular machines propelled by local conforma- 
tional changes. ${ }^{14,15}$ Instead of using molecular mechanics only, another elegant way to induce molecule motion consists of using inelastic tunneling electrons to excite appropriate vibration modes of the molecule. The coupling between electrons and vibronic modes can result in molecule translation, ${ }^{16-18}$ rotation ${ }^{19,20}$ as well as chemical reactions. ${ }^{22-25}$ However, vibrationally activated molecular displacement has, in principle, a large stochastic part rectified by the molecule itself. To benefit from it, a proper molecular design ${ }^{26}$ and/or the use of an external electric field in the tunneling junction are required to impose the direction of motion on a surface. ${ }^{17,27,28}$ Not only restricted to the molecule design, the surface diffusion is also governed the subtle interplay between the molecule adsorption and the underlying surface potential which must be addressed in detail. ${ }^{29-31}$ Such investigations provide fundamental understanding of the surface energy landscape, the electronic and vibrational excitations as well as the diffusion processes of complex molecular structures at surfaces.

Here, we report on the adsorption and diffusion properties of the 4'-(4-Tolyl)-2,2':6',2"'terpyridine, so-called Swiss Nano Dragster, on the Au(111) herringbone structure by combining high-resolution STM/AFM imaging and density functional theory (DFT) calculations. The translation of the molecule is induced by inelastic excitations through electron injections or extractions at $4.7 \mathrm{~K}$. The vibrationally excited molecule also responds to the inhomogeneous electrical field induced by the STM tip in the tunneling junction which allow us to govern the direction of translation. Single-molecules are efficiently displaced all over the reconstruction but show drastic differences of their diffusion at specific elbows acting as pinning centres. The experimental data supported by DFT calculations show the formation at those locations of a coordination complex between the terpyridine unit of the molecule and $\mathrm{Au}$ atoms of the surface which modifies the molecule conformation. Using force spectroscopic measurements, we quantify there an increase of the lateral force threshold required to mechanically move the molecule of $\sim 280 \mathrm{pN}$ corresponding to an increase of the surface diffusion barrier of the molecule of $\sim 100 \mathrm{meV}$ compared to the rest of the surface. 

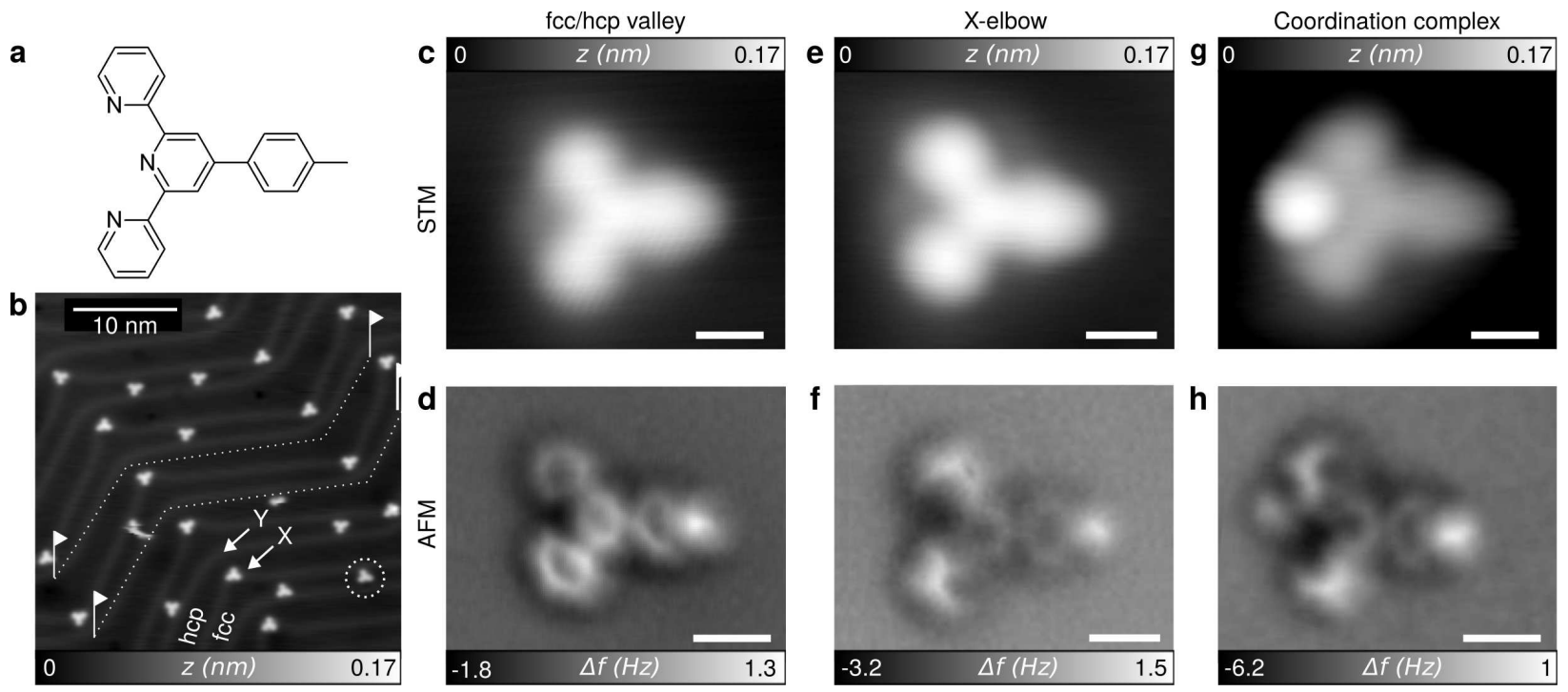

Figure 1: Comparison of The Adsorption Geometries of Single SND Molecules on $\mathbf{A u}(\mathbf{1 1 1})$. a, Chemical structure of the Swiss Nano Dragster (SND) molecule, 4'(4-methylphenyl)-2,2':6',2"-terpyridine. b, STM overview of the adsorbed molecules on $\mathrm{Au}(111)$. The molecules first adsorb at specific elbows of the herringbone structure denoted "X" and then along the valleys of the fcc/hcp reconstruction, $\left(\mathrm{I}_{t}=2 \mathrm{pA}, \mathrm{V}_{\text {tip }}=-150\right.$ $\mathrm{mV}$ ). The dashed lines represent the racetrack. c, STM image of the molecule at the valley and $\mathbf{d}$, the corresponding AFM image. e, STM image of the molecule at the X elbow and $\mathbf{f}$, the corresponding AFM image. g, STM topographic image of an Au coordination complex and $\mathbf{h}$, the corresponding AFM image. Scale bars $=0.5 \mathrm{~nm}$.

\section{Results And Discussion}

Swiss Nano Dragster And The Gold Racetrack: The 4'-(4-methylphenyl)-2,2':6',2"terpyridine molecule, ${ }^{32}$ so-called Swiss NanoDrasgter (SND), has a planar structure composed of three pyridine units and one front 4-methylphenyl unit linked by interannular CC bonds (Figure 1a). The terpyridine moieties act as coordination center favouring the formation of coordination bonds with transition metals via the lone-pairs of the pyridine nitrogens. ${ }^{32}$ On the surface, these specific and localized interactions of the terpyridine are expected to locally enhance the molecule adsorption by interacting with the gold substrate and also respond to the external electrical field of the STM tip. This is slightly in contrast to previous molecular machines which are propelled at surfaces by conformational changes driven by electrons from the STM tip. ${ }^{14,15}$ The front tolyl unit weakly physisorbs on gold by 

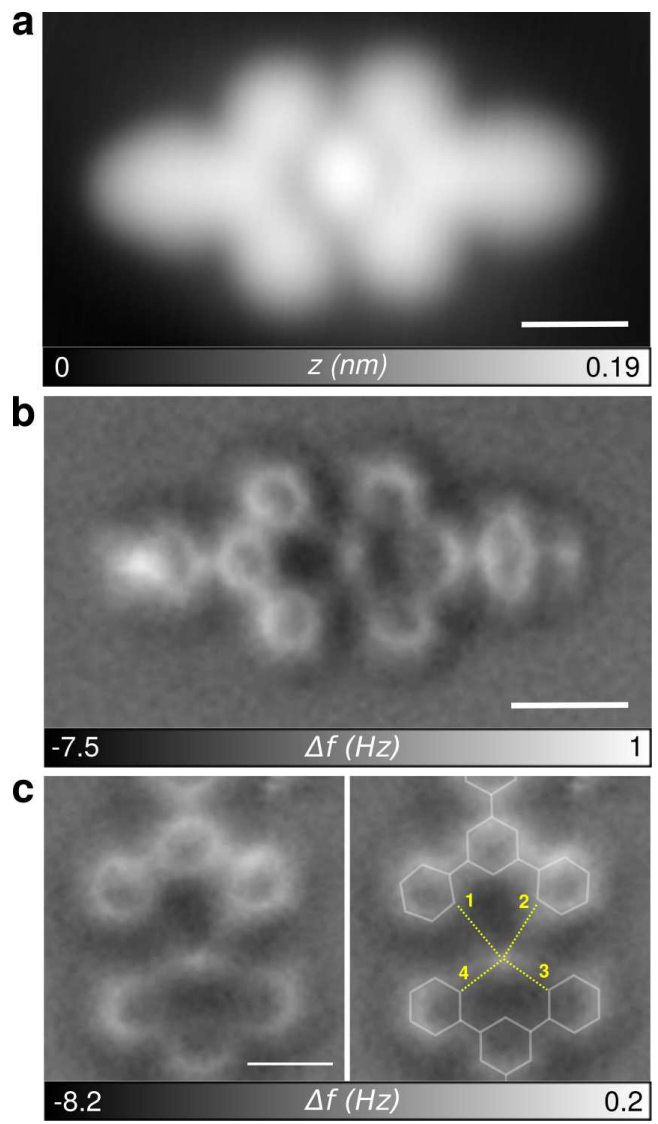

Figure 2: Adsorption Geometry of The SND-Au-SND Complex on Au(111). a, STM topographic image of the SND-Au-SND coordination complex $\left(\mathrm{I}_{t}=2 \mathrm{pA}, \mathrm{V}=-150\right.$ $\mathrm{mV}$ ) and $\mathbf{b}$, The corresponding AFM image with a CO-terminated tip. c, Close-up AFM image of the coordination center revealing the Au-molecules bonding. The yellow dashed lines symbolizes the coordination bonds expected for such planar coordination geometry. The bond lengths are $1=315 \mathrm{pm}, 2=330 \mathrm{pm}, 3=223 \mathrm{pm}$ and $4=224 \mathrm{pm}$. Scale bars $=$ $0.5 \mathrm{~nm}$.

van der Waals interactions. ${ }^{10}$ This unit acts as front spoiler of the vehicle since it is expected to balance the friction forces during translation on the gold surface.

The $\mathrm{Au}(111)$ surface is characterized by a hexagonal arrangement of atoms with a herringbone reconstruction. The reconstruction is composed of valleys, ridges and elbows that result from alternating face-centered-cubic (fcc) and hexagonal-close-packed (hcp) regions (see STM atomic resolution in Supp. Info. Figure S1). Previous experimental works showed adsorption peculiarities of atoms and molecules the two coexisting elbow sites, denoted $X$ and $Y$ in Figure 1b. ${ }^{33,34}$ During the NanoCar Race, the competitors had to realize three 
straight lines and two curves along this reconstruction as depicted in Figure 1b. Understanding the complexity of the $\mathrm{Au}(111)$ surface potential with respect to the molecular vehicle is thus a fundamental challenge to foreseeing its adsorption as a single-molecule and its displacements.

Adsorption Geometries of The SND Molecules on Au(111). A first prerequisite of the NanoCar Race was to obtain single-molecules on a Au(111) sample kept at room temperature from thermal deposition in ultra-high vacuum. For the race purpose, single-molecules were indeed preferable instead of assemblies to facilitate manipulations. Upon deposition of the SND molecule in that conditions, a clear hierarchy of the adsorption is observed since single-molecules first sit at the $X$ elbows (Figure 1b) whereas the adjacent $Y$ elbows always remain free. After saturation of those $X$ elbows, single SND molecules are then found located along the fcc and hcp valleys as shown by the dashed circle of Figure 1b. Figures 1c-h report all the adsorption geometries observed at the surface by STM/AFM imaging at $4.7 \mathrm{~K}$ with CO-terminated tips. Figures 1c and d show typical STM/AFM images of the SND molecule at the valley position. The AFM image (Figures 1d) reveals that the molecule adopts a flat conformation where the bright protrusion corresponds to the tolyl unit and the three other rings are the pyridine units. Figure 1e shows a STM image of the molecule located at the $X$ elbow which does not reveal drastic differences in comparison to the valley site (Figures 1c). The corresponding AFM image (Figure 1f) however shows a clear conformational change consisting of a slight rotation of the peripheral pyridine units along the $\mathrm{C}-\mathrm{C}$ bonds. This geometrical adaptation in comparison to the flat conformation suggests a variation of the molecule-surface interactions, that arises from the formation of coordination bonds between the pyridine units and gold atoms of the underlying $X$ elbow site.

This interpretation is further supported by studying other coordination complexes spontaneously formed at the surface between SND molecules and Au adatoms. Figures $1 \mathrm{~g}$ and h shows a STM image of a Au-SND complex and the corresponding AFM image obtained 
with CO-terminated tips which reveals the presence of the Au adatom at the proximity of one pyridine center. The rotation of the peripheral pyridine units is observed due to the complex formation in analogy to the elbow case. However, in comparison with the Au-SND complex, the AFM image of the elbow conformation (Figure 1f) does not show the presence of the Au adatom. Recent works have also reported similar conformational adaptation of molecules by $\mathrm{AFM}$ imaging upon complex formation with $\mathrm{Au}$ or $\mathrm{Cu}$ adatoms. ${ }^{37,38}$ It was shown that, although the molecule also modifies its geometry, the adatom involved in the complex formation does not always have a clear signature in the AFM data depending on its position with respect to the molecule plane. ${ }^{38}$ We thus assume that a similar effect arises in Figure 1f since the Au atom in that case is pulled from the surface ( $X$ elbow) and not a surface atom in the molecule plane.

SND-Au-SND complexes are also observed at the surface as shown in Figure 2. The terpyridine units of both SND molecules are rather planar in that case (Figure 2b). A closer look at the coordination center in Figure 2c suggests an increase of the number of coordination bonds of the SND-Au-SND case to four (depicted by yellow lines) compared to the the Au-SND complex assumed to be three. We also estimated the Au-molecule bond lengths in the coordination center that vary from 220 to $330 \pm 25 \mathrm{pm}$. In contrast to references, ${ }^{37,38}$ the coordination bond lengths are much larger in our case but in agreement with values found in porphyrine or phthalocyanine macrocycles $(\sim 205 \mathrm{pm}$ and $190 \mathrm{pm})$. As a result, we think that the coordination geometry of the SND-Au-SND complex evolves from a trigonal pyramidal in the Au-SND complex case to a rather square planar geometry in analogy to porphyrin macrocyles. This might explain the planarization of the pyridine units in the SND-Au-SND case similar to porphyrin macrocycle.

To better understand the adsorption of the single SND molecules and their electronic properties, we intensively performed DFT calculations on an unreconstructed gold surface (see Methods). Figure 3 shows the relaxed structure of the flat-lying SND molecule (Figure 3a) and the Au-SND complex (Figure 3d). The simulated AFM image (Figure 3b) 

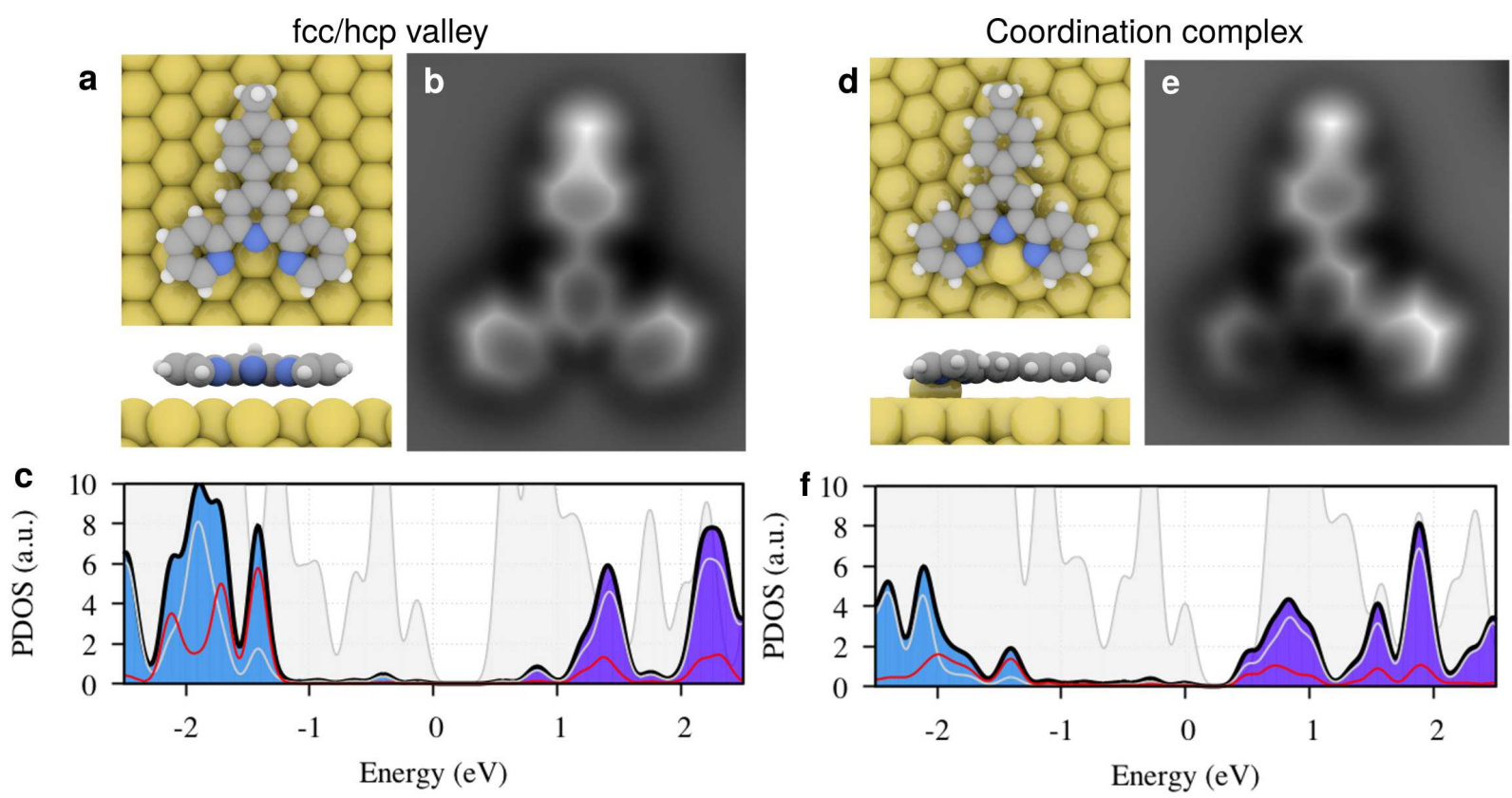

Figure 3: Flat-Lying Molecule Versus Au-SND Coordination Complex. a, Top and side views of the adsorption geometry of the molecule at the valley location obtained by DFT calculations. b, Simulated AFM image from the relaxed structure of a. c, Projected density of states (blue). d, Top and side views of the absorption geometry at the Au-molecule coordinate obtained by DFT optimization using VASP. e, Simulated AFM image revealing the tilting of the pyridine end-groups due to coordination. f, Projected density of states of the system. In $\mathbf{c}$ and $\mathbf{f}$, the contribution of the gold is shown by the grey shaded areas, carbon by grey lines and nitrogen by blue lines, respectively. The total PDOS of the molecule is shown as blue and purple shaded area for the occupied and unoccupied states.

obtained from the calculated geometry with the probe particle model of reference ${ }^{39}$ is in excellent agreement with the experimental data (Figure 1d). Note that several local minima are also possible for such flat conformation in terms of position with respect to the surface lattice (see also Supp. Info. Figure S3). The simulated AFM data of those other configurations are always in agreement with the experimental AFM data. Figure 3c shows the projected density of states of the flat conformation with the occupied and unoccupied molecular states coloured in blue and purple, respectively. The gray area refers to the PDOS of the gold surface. The red and gray curves represent the PDOS of the nitrogen and carbon atoms of the molecules, respectively. The highest occupied molecular state (HOMO) is located at $\sim-1.25 \mathrm{eV}$ and the lowest unoccupied state (LUMO) at $\sim 0.8 \mathrm{eV}$ corresponding to a HOMO-LUMO gap of $2.05 \mathrm{eV}$. 

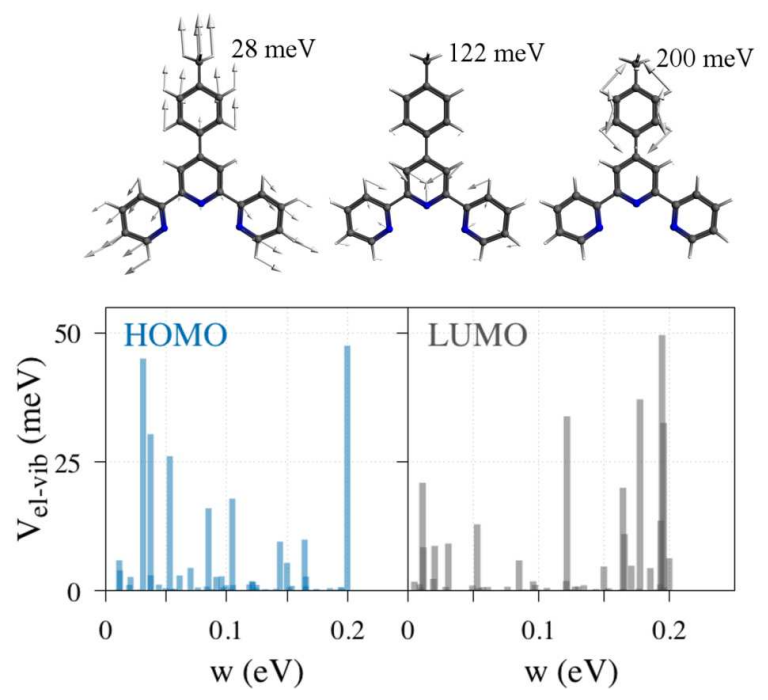

Figure 4: Vibration Modes And Electrons-Phonon Coupling of The "Flat" Molecule. The top images shows the vibration modes of the SND molecule structure. The graphs shows electrons-phonon coupling of the flat SND molecule though HOMO (blue) and LUMO (gray) excitations, respectively.

Regarding the calculated Au-SND complex of Figure 3d, the pyridine nitrogens of the Au-SND complex are strongly coordinated with the surface gold atom which leads to the rotation of the pyridine groups. This tilt is clearly observed in the simulation of the AFM picture (Figure 3e) in agreement with the experimental observations (Figures 1f and h). As aforementioned, the $\mathrm{Au}$ adatom in the AFM simulation is not visible as in the experimental data which can be imputed to its position slightly below the molecule plane in the simulation compared to the experiment. Nevertheless, the DFT calculations confirm the coordination bonds between the lone pair electrons of the terpyridine units and the Au surface adatom, either lifted from the surface or in the molecule plane, that drastically modify the molecule geometry compared to its flat conformation. Figure $3 \mathrm{f}$ shows the projected density of states of such Au-SND complex with the occupied and unoccupied molecular states coloured in blue and purple, respectively. Compared to Figure 3c, the LUMO of the Au-SND complex is shifted towards the Fermi level $(\sim 0.5 \mathrm{eV})$ whereas the HOMO position remains rather unchanged $(\sim-1.25 \mathrm{eV})$. Upon complexation, the HOMO-LUMO gap decreases to $\sim 1.75$ eV. 

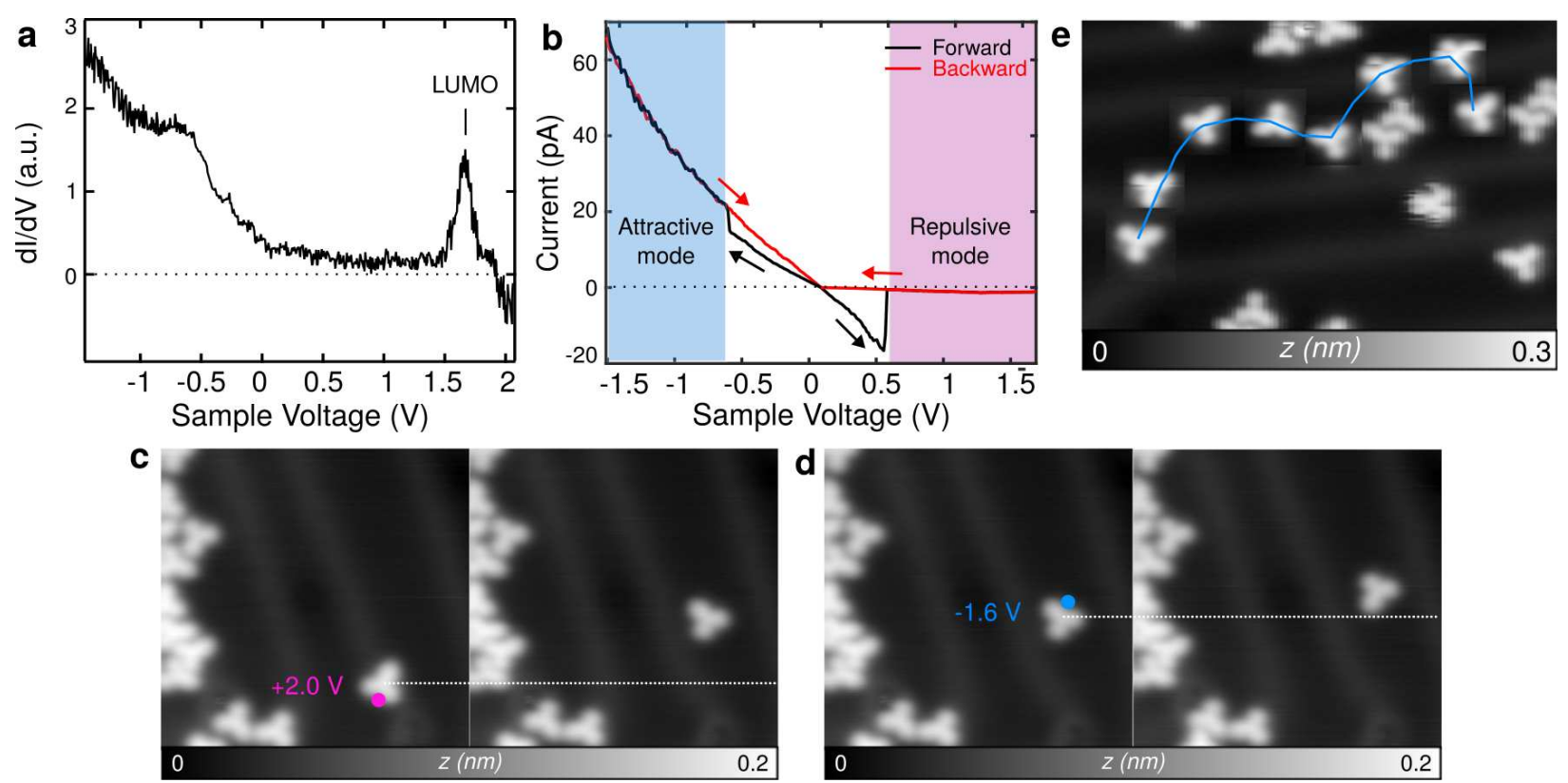

Figure 5: Single-Molecule Displacements Induced by Inelastic Electrons. a, Experimental differential conductance, $d I / d V$, of the SND molecule at valley position. $\mathbf{b}$, Current-voltage spectra, $I(V)$, obtained at $4.7 \mathrm{~K}$ with a gold-coated tip showing the minimum threshold voltages $( \pm 500 \mathrm{mV}$ ) inducing the molecule motion. Depending on the polarity, the molecule is either repelled (negative tip sample) or attracted (positive tip voltage) from the tip position where the pulse is applied. c, STM images showing controlled translations using the repulsive mode $\left(\mathrm{V}_{\text {sample }}=-2.0 \mathrm{~V}\right)$ and the attractive mode $\left(\mathrm{V}_{\text {sample }}=\right.$ $+1.6 \mathrm{~V}),\left(\mathrm{I}_{t}=2 \mathrm{pA}, \mathrm{V}=150 \mathrm{mV}\right)$. The red and yellow dots refer to the tip position prior to the excitation at the corresponding voltages. g, Snapshots of an elaborated manipulation path across ridges and valleys (in blue) using the attractive mode.

Actuation by Inelastic Tunneling Electrons. Knowing the various molecular geometries, our aim during the NanoCar Race was to displace individual molecules over long distances in a repeatable manner using inelastic tunneling electrons from the STM tip. Among all reported geometries, we targeted the flat conformation expected to have the smallest adhesion and which is the most encountered at the $\mathrm{Au}(111)$ surface. Through injection or extraction of tunneling electrons, the excitation of the molecule vibration modes is expected to play a fundamental role in the molecule motion. ${ }^{17-21}$ To better understand this aspect of the process, the coupling strengths between the charge carriers and the vibration modes (Figure 4) were extracted from numerical calculations of the flat conformation (Figure 3thata and see also Methods). Different vibration modes with energies up to $0.2 \mathrm{eV}$ present a sig- 
nificant coupling with both, electrons and holes.

Figure 5a shows a typical differential conductance $d I / d V$ spectra of the molecule on $\mathrm{Au}(111)$ acquired at $4.7 \mathrm{~K}$ with a gold-coated tip obtained by a gentle indentation into the bare surface. For positive sample voltages, a sharp resonance at $\sim 1.6 \mathrm{~V}$ is observed which is assigned to the LUMO resonance peak of the molecule and in good agreement with the DFT calculations (Figure 3c). At negative sample voltages, the assignment of the HOMO molecule frontier orbitals is rather difficult due to the important contribution of underlying gold electrons. According to the calculations (Figure 3c), the broad resonance peak has a shoulder at $\sim-0.5 \mathrm{~V}$ coinciding to gold states (Supp Info. Fig. S2) and only a small contribution from the molecule states between $-1 \mathrm{eV}$ and the Fermi level is present.

Steering And Technical Characteristics of The SND on Gold. Figure 5b shows a typical $I(V)$ trace acquired above the molecule to induce its displacement with a gold-coated tip obtained by gentle indentation into the gold surface at $4.7 \mathrm{~K}$. Note that the reproducibility of this curve as well as the later described manipulation process is intrinsically bound to the tip quality and thus its preparation described in the Methods section. A successful event is experimentally observed as an abrupt jump in the curve. Comparing the current variation between the forward (black) and backward (red) $I(V)$ curves reveals that the molecule is either repelled from the tip position for sample voltages $\leq 500 \mathrm{mV}$ or attracted below the tip for negative sample voltages $\leq-500 \mathrm{mV}$. The minimum threshold voltage to induce the molecule motion is $\pm 500 \mathrm{mV}$ which is above the energy of the fundamental vibrational modes of the molecule (Figure 4). Although the electronic excitation is not in resonance with those fundamental vibronic modes, they might be still accessible through indirect tunneling processes using higher voltages. Interestingly, the observation of the direction of displacement depending on the voltage applied in the tunnelling junction suggest a coupling of the electric field with the excited vibration modes in analogy to reference. ${ }^{27}$ Indeed, we observe 
a clear difference of the direction of displacements depending on the tip voltage as shown in the graph Figure 5b. More precisely, negative tip voltage (or positive sample voltage) leads to a "repulsive mode" meaning that the molecule is always repelled from the tip position (red area in Figure 5c). For positive tip voltages (i.e. negative sample voltages), the molecule is instead attracted towards the tip position during the pulse (Figure 5d). This mode is thus referred to the "attractive mode". It has, compared to the repulsive mode, the advantage of allowing the control of the direction of displacement which is strictly determined by the tip position prior to the excitation. Although the mean free path of the molecule per excitation is much smaller with the attractive mode than the repulsive mode $(\sim 0.6-0.8 \mathrm{~nm}$ versus $2-3$ $\mathrm{nm}$ ), this mode is preferred to achieve long manipulation path (Figure 5e) since it allows the steering control.

To prove the high control the manipulation procedure, Figure 5e shows a complex path realised over the herringbone reconstruction using the attractive mode (see also supplementary movie). A total distance of $\sim 27 \mathrm{~nm}$ was achieved in $90 \mathrm{~min}$ using $\sim 45 \mathrm{I}(\mathrm{V})$ sweeps corresponding to an average distance per excitation of $\sim 0.6 \mathrm{~nm}$ and a maximum speed of about 16-20 nm.h ${ }^{-1}$. Although the voltage sweep time above the molecule required for its excitation is in the order of 1-2 seconds, the limitation of the speed relies on the number of STM images acquired between each excitation, their acquisition time $\left(\sim 3\right.$ min.image $\left.{ }^{-1}\right)$ as well as their resolution $\left(256 \times 256\right.$ pixels $\left.^{2}\right)$. Improvements can be clearly done regarding these aspects but are not in the scope of the present work.

The molecule translation is induced for a minimum tunneling current of $\mathrm{I}= \pm 20 \mathrm{pA}$ at voltages of $\sim \pm 500 \mathrm{mV}$ (Figure 5b). This corresponds to an minimum electrical power delivered to the tunneling junction, defined as $\mathrm{P}_{\text {elec }}=\mathrm{U} \times \mathrm{I}$, of $\sim 10 \mathrm{pW} \cdot{ }^{29,30}$ While keeping similar current values, larger voltages of $\sim \pm 1.8 \mathrm{~V}$ are however preferable to increase the success rate of the manipulation process as discussed in reference. ${ }^{27}$ Typical powers $\mathrm{P}_{\text {elec }}$ are in the order of $36 \mathrm{pW}$. Too large excitation power, e.g. $\geq 300 \mathrm{pW}(\mathrm{U}= \pm 3 \mathrm{~V}, \mathrm{I}=100 \mathrm{pA})$, leads to the destruction of the molecule as shown in Figures 6a and b. This corresponds 


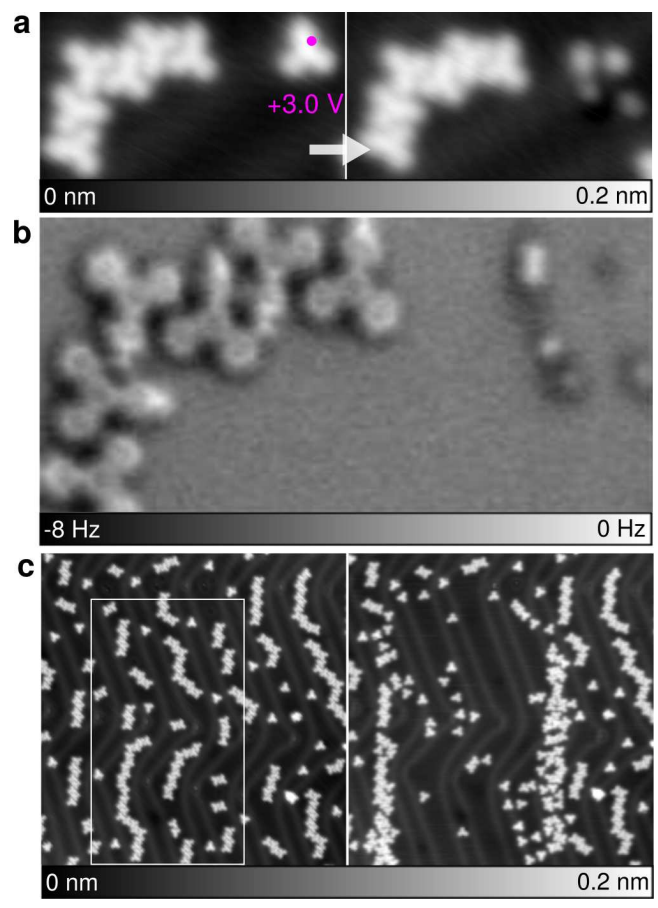

Figure 6: Destruction of SND Molecule Vehicle And Preparation of The Racetrack. a-b, "Accident" that can occur when using too high excitation power to displace the SND molecule. Upon trapping at elbows or close to molecule assemblies, the electronic excitation requires higher voltages. For voltages of $\pm 2.8 \mathrm{~V}$, the molecule structure can be destroyed as revealed in the STM and corresponding AFM image with a CO-terminated tip b. c, Successive STM images with a $\mathrm{Au}$-coated tip showing the removal of surrounding SND molecules from the gold racetrack, $\left(\mathrm{I}_{t}=2 \mathrm{pA}, \mathrm{V}=100 \mathrm{mV}, \mathrm{R}=50 \mathrm{G} \Omega\right)$. An STM image is acquired at the white rectangle with a smaller tunneling resistance to wipe away the molecules $\left(\mathrm{I}_{t}=250 \mathrm{pA}, \mathrm{V}=100 \mathrm{mV}, \mathrm{R}=400 \mathrm{M} \Omega\right)$.

to the maximum electrical power of the tunnel junction that the molecule can theoretically hold. We also emphasize that these values do not correspond to the effective power required to excite the molecule displacement since most of the electrons tunnel elastically through the molecule (without triggering any inelastic molecular excitations) and dissipate into the substrate.

Experimentally, the SND molecule can be efficiently translated along both fcc and hcp valleys as well as across ridges. Short curves are possible allowing the overtaking of singlemolecules and their assemblies as shown in Figure 5e. However, to complete the long distance of the NanoCar Race racetrack, further strategies were explored to clean the molecules from the racetrack. Although most of the single SND molecules can be removed using the method 


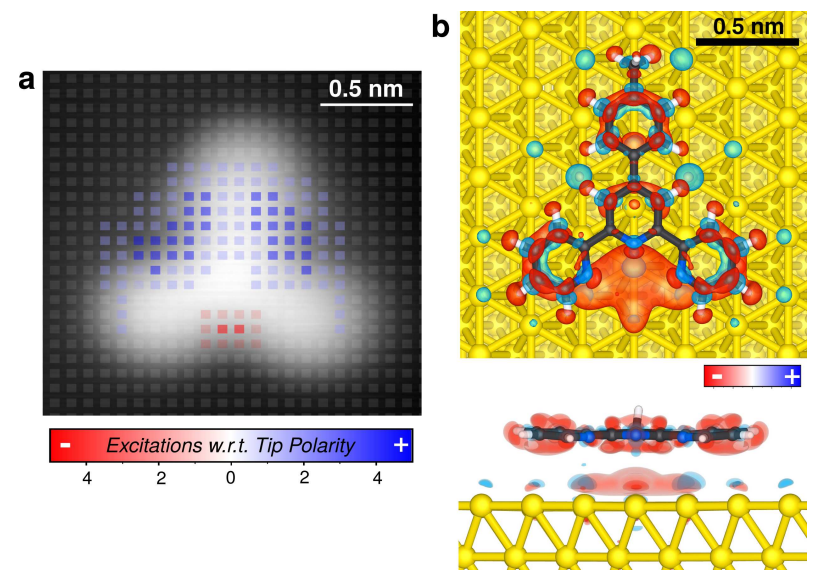

Figure 7: Comparison Between The Experimental Excitation Map And The Charge Density Difference Obtained From DFT Calculations. a, Excitation map of the molecule as a function of the tip position and polarity. The blue and red pixels refers to positive tip voltages (attractive mode) and negative tip voltages (repulsive mode) of \pm 1.8 $\mathrm{V}$, respectively. $\mathbf{b}$, Modification of the electronic density of the molecule-surface complex upon adsorption. The red and blue area corresponds to an excess and lack of electrons, respectively.

described above, this technique cannot be employed in case of self-assemblies. For that, the molecules must be wiped away as shown in Figure 6c using STM scans with smaller tunneling resistance compared to the stable scan conditions.

A Field-Assisted Vibrationally-Excited Propulsion: The control of the steering is not explained by only considering inelastic processes involving the molecule vibrations since this does not impose in principle a direction of motion. As reported in reference, ${ }^{27}$ the vibrationally-excited molecule might also respond to the local electric field of the STM junction. To better understand this aspect, we built up an excitation map of the SND molecule as a function of tip position and polarity. Each successful molecule manipulation was reported on a grid of $25 \times 25$ pixels $^{2}$ above the molecule shown in Figure 7a. The blue and red pixel colors refer to successful translations using positive tip voltages (attractive mode) and negative tip voltages (repulsive mode) of $\pm 1.8 \mathrm{~V}$, respectively. The pixel color intensity represents the number of successful hopping events where a total number of 200 manipulations are reported. Since the tip quality has an important role in the manipulation 
process, the reproducibility of its conditions was ensured by gentle indentations of $\sim 1 \mathrm{~nm}$ into the bare gold at $4.7 \mathrm{~K}$. That way, symmetric gold-coated tip apexes were systematically produced for the manipulations. We avoided all undefined tip apexes in the map statistics which can lead to unexpected molecule displacements. Note finally that the molecule excitations as well as the indentation procedure were successfully reproduced in the four-probe microscope hosting the NanoCar Race.

The repulsive mode (red pixels) requires to excite the molecule precisely at the location of the terpyridines whereas the tip positioning for the attractive mode (blue pixels) is more delocalized at the surrounding of the molecule. For the repulsive mode, i.e. for electron injections, the hopping motion has a random direction and more difficult to activate experimentally (only 20 counts). This mode was not further explored because the direction of the molecule motion is not controlled in contrast to the attractive mode. In this last mode, the direction of translation as well as the final position of the molecule correspond to the pixel position before excitation (blue pixels in Figure 7a), i.e. the tip position. Based on this map, we steered our vehicle during the training and the NanoCar Race over paths longer than $100 \mathrm{~nm}(\sim 160$ excitations).

The polarity-dependent molecule translations suggest the response of the molecule to the electric field of the tunnel junction. ${ }^{27}$ To clarify this point, we compute using DFT calculations the charge density difference of the adsorbed SND molecule in the flat conformation (Figure $7 \mathrm{~b}$ ). The red and blue area correspond to an excess and a lack of electrons, respectively. The molecule is negatively charged at the surface $\left(q_{m o l}=-0.36 \mathrm{e}\right)$ which induces charge depletion areas in the underlying gold lattice (blue areas). An important electron accumulation is observed at the terpyridine coordination center. Assuming this location to be a single-point charge compared to the rest of the molecule, the control of the direction is intuitively explained as the response of the charge at the terpyridine center to the electric field of the tip. The scattering of the blue pixels around the molecule (Figure 7a) coincides with the average translation distance obtained with the attractive mode $(\sim 0.6-0.8 \mathrm{~nm})$. 
This observation suggest that, although the molecule is sensitive to the tip electric field, exciting the vibration mode of the molecules through electron extraction is mandatory to allow its translations. More precisely, applying large field off the molecule without tunneling though any molecular orbitals will not induce molecule motions. As introduced by Ohara et al. ${ }^{27}$ we conclude that the propulsion consists in a field-assisted vibrationally-excited molecular motion. However, we observed that this scenario is not valid anymore upon complex formation of the SND molecule with surface Au atoms. there, the chemical binding strongly modifies the charge character of the molecule, the vibration mode as well as its diffusion properties. ${ }^{28}$ This aspect related to the position of the molecule with respect to the surface is a key issue that we had to experimentally explore to achieve the curves along the gold reconstruction.

\section{Site-Dependent Lateral Force Required to Move The Molecule : Effect of the}

Elbow. To quantify the local variation of the molecule diffusion barrier, force spectroscopic measurements were conducted to extract the lateral force and potential energy required to move the SND molecule over the gold reconstruction. ${ }^{11,40-42}$ Figures $8 \mathrm{a}$ and b shows successive frequency shift versus $x$ distance curves, $\Delta f(x)$ (grey curves) acquired from left to right with a metallic tip at $4.7 \mathrm{~K}$ above molecules located at elbow and valley sites while reducing the tip-sample distance by $300 \mathrm{pm}$. The red curves refer to the last trace inducing the molecule displacement and the arrows correspond to the threshold lateral force potential barrier required to activate the molecular motion (Figures $8 \mathrm{c}$ and $\mathrm{f}$ ). The molecule at the $X$ elbow (Figure 8a) is pushed by the tip over several atomic sites as revealed by the typical stick-slip of $\Delta f$ curve which is related to the $\mathrm{Au}(111)$ lattice constant. ${ }^{11-13}$ The molecule at valley position (Figure 8d) is pulled by the tip with a less pronounced stick-slip pattern which suggests a lower tip-molecule interactions. The resulting potential and lateral force fields for the $X$ elbow and valley site are plotted in Figures 8b-c and e-f, respectively. The threshold lateral force required to move the molecule at the $X$ elbow is $\mathrm{F}_{L}^{X e l b o w} \sim-400 \mathrm{pN}$, 

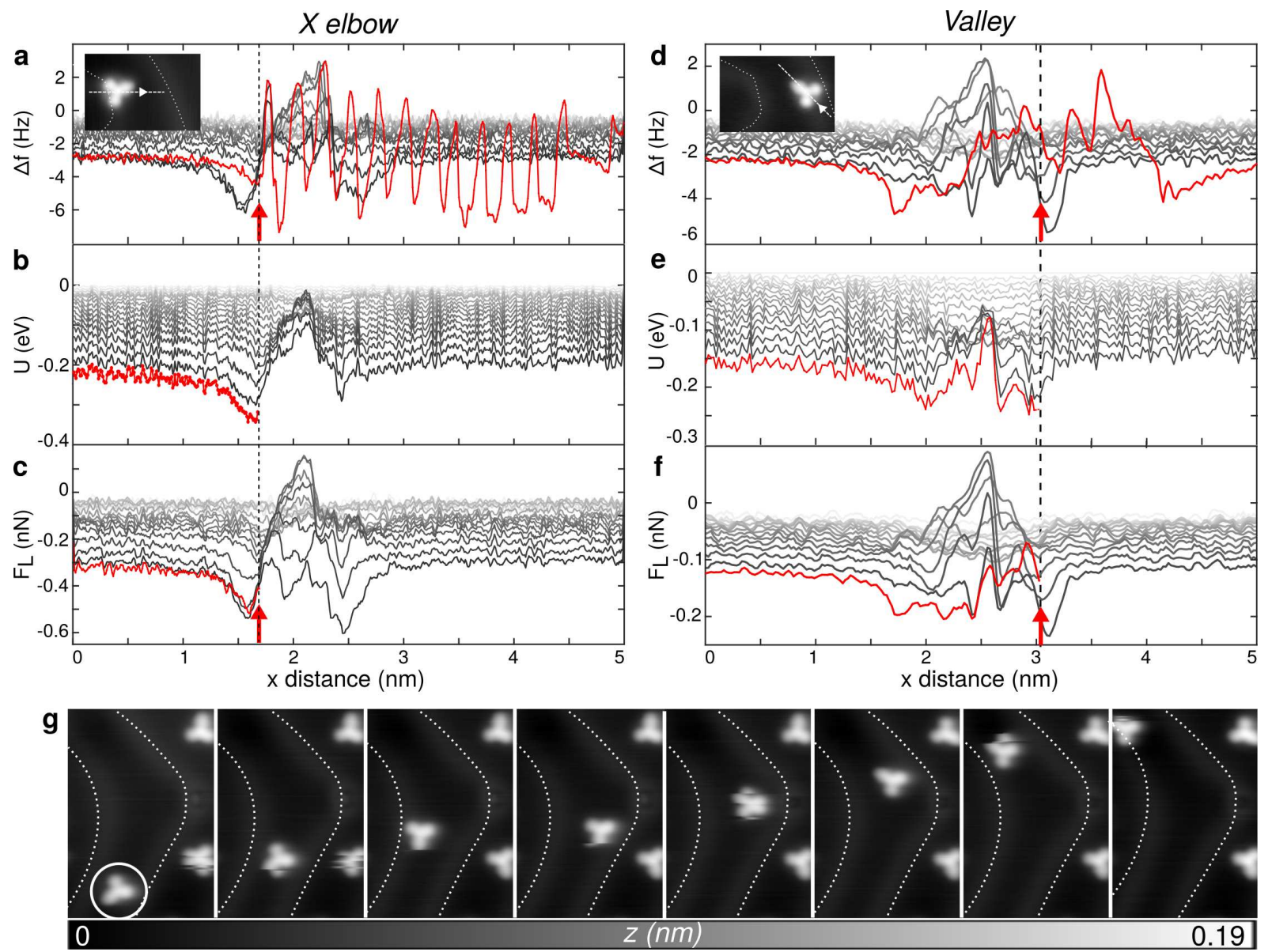

Figure 8: Lateral Force to Move The Molecule : Elbow vs. Valley. a, Successive $\Delta f(x)$ traces acquired above a SND to mechanically induce its motion from an elbow position. b-c, Extracted potential $U(x)$ and lateral forces $F_{x}(x)$ from a. The displacement occurs at the red arrow in $\mathbf{c}$ and $\mathbf{f}$ and corresponds to an attractive lateral force of $400 \mathrm{pN}$ and $120 \mathrm{pN}$, respectively. $\mathrm{d}, \Delta f(x)$ traces acquired above a SND to mechanically induce its motion along the $\mathrm{Au}(111)$ valley. e-f, Extracted potential and lateral forces from d. The motion is obtained at the red arrows and corresponds to an attractive lateral force of $\approx$ 170 pN. g, Successive STM images showing how a curve is realized with the SND molecule (circle) by avoiding the $X$ elbow of the gold reconstruction, $\left(\mathrm{I}_{t}=5 \mathrm{pA}, \mathrm{V}_{\text {tip }}=-150 \mathrm{mV}\right)$.

whereas, at the valley site, this value increases to $\mathrm{F}_{L}^{\text {valley }} \sim-120 \mathrm{pN}$. This site-dependent variation of $\sim 280 \mathrm{pN}$ is the direct consequence of the coordination bonds formed with the surface atoms of the $X$ elbows (Figure 1f) in contrast to others adsorption sites. Comparing the potential fields (Figures 8b and e), the measured diffusion barrier needed to displace the molecule between neighbouring atomic sites is $\sim 330 \mathrm{meV}$ for molecules at the $X$ elbow and $\sim 230 \mathrm{meV}$ for the valley sites, respectively. These values are in remarkable agreement with 


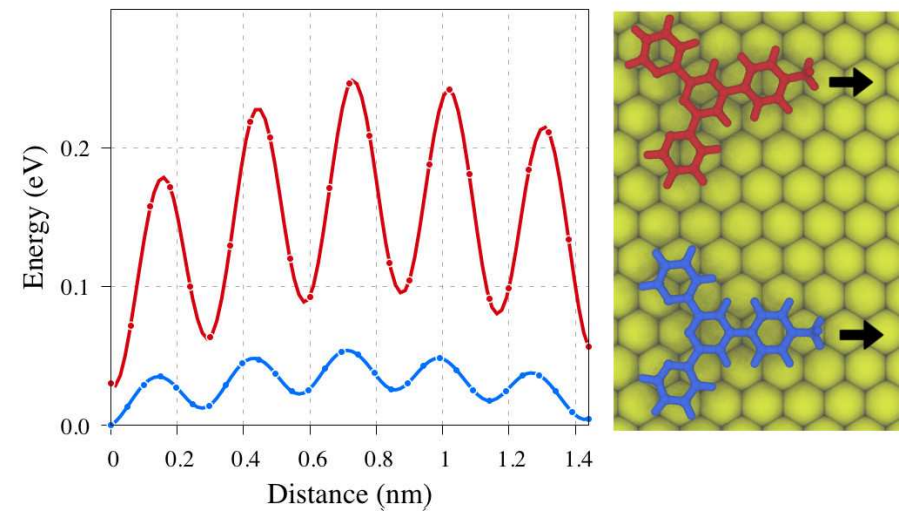

Figure 9: Diffusion Energy Barrier of The Flat SND Molecule. Energy landscape for the diffusion along a straight path for two distinct orientation of the molecule on an unreconstructed gold surface.

previous DFT calculations performed for other organic molecules on gold. ${ }^{30,43}$

The energy landscapes for diffusion of two adsorption sites of the flat conformation were also estimated via DFT calculations on an unreconstructed gold surface (see Methods). Depending on the initial orientation of the molecule on the surface (right inset of Figure 9), the energy values varies between $60 \mathrm{meV}$ to $140 \mathrm{meV}$. The potential landscape shows values in the same order of magnitude than the experimental data (Figure 8e) as well as a very small modification of the conformation during translation. In close agreement with the experimental data, the energy landscape has also a modulation with a periodicity of $0.288 \mathrm{~nm}$ which corresponds to the $\mathrm{Au}-\mathrm{Au}$ interatomic distance. Superimposed to these oscillations, a longer modulation with a periodicity of $1.44 \mathrm{~nm}$ is also observed and corresponds to five $\mathrm{Au}-\mathrm{Au}$ bond. This arises from the interaction of the molecule with the sub-surface gold layers.

NanoCar Race. During the preparation of the NanoCar Race, we learnt that the important aspects of the competition such as the molecule adsorption, its excitation and steering with the STM as well as the surface peculiarities are interconnected parameters that must be understood as an ensemble to achieve navigation over long distances with precision. The molecule design, its size and weight are naturally the key features to foresee the driving 


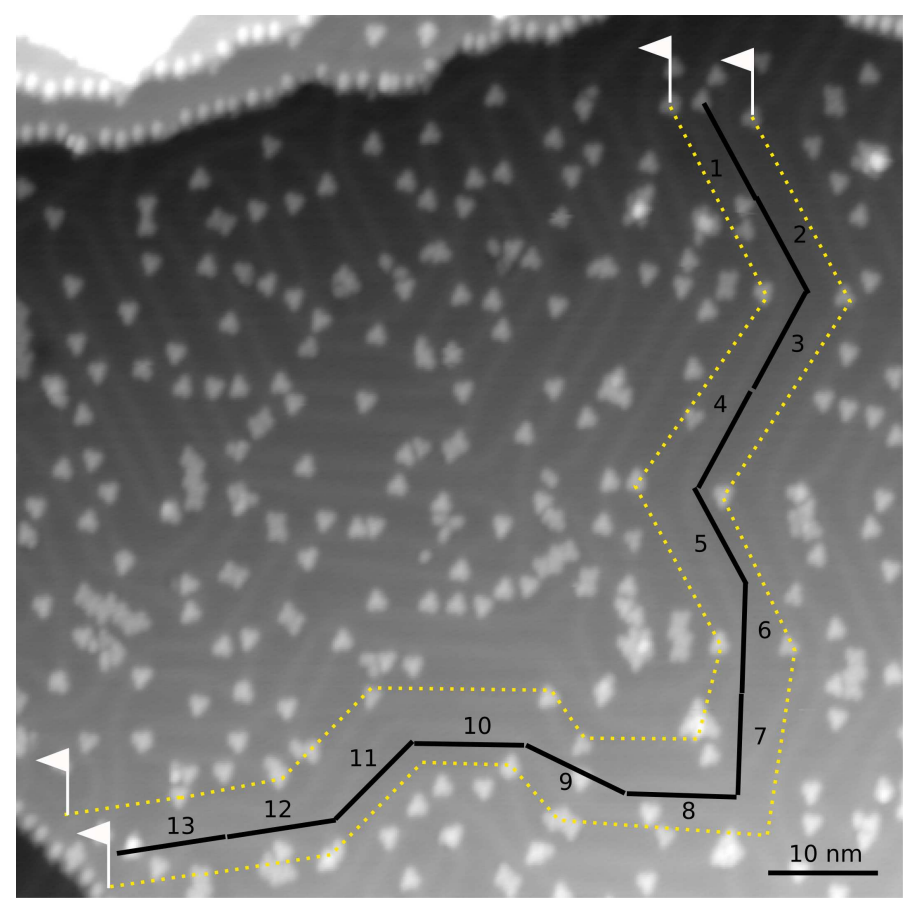

Figure 10: The Racetrack During The NanoCar Race. The track consists of nine straight lines and nine curves for a length of $133 \mathrm{~nm}$. Each segment (black lines) is 10$\mathrm{nm}$ long. A total of 6 hours and 30 min was required to complete it using four molecules and $\sim 250$ excitations. Before the race, several molecules were removed from the track by tip-induced manipulations and parked along the racetrack. $\left(\mathrm{I}_{t}=5 \mathrm{pA}, \mathrm{V}_{\text {tip }}=-250 \mathrm{mV}\right)$

behaviour at the surface. As shown in this work, the host surface must be also taken into account to control the adhesion forces at the molecular scale.

During the NanoCar Race, we completed our 133-nm gold-racetrack (dashed yellow lines in (Figure 10) in 6 hours and 30 min with more than 250 excitations and ranked first. We used a total of four molecules to finish the whole distance for an average speed of $20 \mathrm{~nm} \cdot \mathrm{h}^{-1}$. This speed was mainly limited by the number of intermediate STM images required to follow the complex path and to avoid crashes into obstacles of the track. To reduce the obstacles, we also removed prior to the race several molecules from the track by tip-induced manipulations and parked them aside. Only molecules in the flat-lying conformation (Figure 1d) were manipulated using the attractive mode, whereas all other configurations were left at their adsorption position. From all participants, the SND molecule, composed of 43 atoms and an atomic weight of $323.9 u$, was the smallest nanocar. Knowing that typical powers of 
$\sim 100 \mathrm{pW}$ were employed during the race to electrically excite the molecule with the STM tip, we estimate its power-to-atomic-weight ratio to $0.3 \mathrm{pW} \cdot \mathrm{u}^{-1}$ on gold. To facilitate the comparison of the performance of the molecular vehicle operated on common surfaces, we invite future concept cars to use such standard which is independent of the molecule size and weight.

\section{Conclusion}

To summarize, we report our experiences of the controlled manipulations of the Swiss Nano Dragster over the gold herringbone structure in the preparation of the NanoCar Race. Among all the molecule geometries characterized by STM and AFM imaging and coexisting at the gold surface, the controlled diffusion of SND molecule is readily induced for the flat lying SND molecule at valleys. The propulsion consists of the excitation of the molecule vibration modes by electron injection or extraction. Its steering is further governed by the electric field applied to the tunneling junction by the tip. The "field-assisted vibrationally-excited" motion is fully controlled and highly repeatable all over the herringbone reconstruction, except at the elbows acting as pinning sites. Indeed, these locations promote the formation of a coordination complex between the molecule and surface atoms which modifies the intrinsic diffusion properties of the molecule. Using force spectroscopy, we quantify an increase of the lateral force required to move the molecule at this peculiarities of $\sim 280 \mathrm{pN}$ compared to the rest of the surface, corresponding to an increase of the diffusion barrier of $\sim 100 \mathrm{meV}$. Fundamentally, this study underlines the pivotal role of the structure and local reactivity of the gold reconstruction into the adsorption and diffusion properties of surface adsorbates at the atomic scale. This might have further implications for the proper design of molecular machines capable of transporting single-atoms at surfaces with optimized friction properties. 


\section{Methods}

Sample Preparation. $\mathrm{Au}(111)$ single crystals were cleaned by several sputtering and annealing cycles in ultra high vacuum. The 4'-(4-methylphenyl)-2,2':6',2"-terpyridine was molecules were evaporated from a quartz crucible onto the sample kept at room temperature in ultra-high vacuum.

STM/AFM Experiments. The experiments at the University of Basel were carried out at $4.7 \mathrm{~K}$ with an Omicron GmbH low-temperature STM/AFM operated with a Nanonis RC5 electronics. We used commercial and home--made tuning fork sensors in the qPlus configuration $\left(f_{0}=26 \mathrm{kHz}, Q=10000-25000\right.$ at $5 \mathrm{~K}, A=50 \mathrm{pm}$, nominal spring constant $\left.1800 \mathrm{Nm}^{1}\right)$. Unless mentioned, the AFM images were acquired at constant-height with CO-terminated tips. The scanning tunneling spectroscopy was acquired with the lock-in technique $\left(U_{\bmod }=\right.$ $10 \mathrm{mV}, f_{0}=600 \mathrm{kHz}$ ). The experiments at the CEMES were performed with a four-probe low temperature microscope from Omicron $\mathrm{GmbH}$.

Tip Preparation. To obtain symmetric and metallic tips in a reproducible way, we used gentle indentations of $\sim 1.0 \mathrm{~nm}$ into the bare gold substrate at $4.7 \mathrm{~K}$. That way, symmetric gold-coated tip apexes were systematically produced and used for the manipulations. The CO terminated tips were obtained by picking up a single CO molecule adsorbed on the gold surface using a $Z$ spectroscopic curve and used for the AFM images.

Excitation Map. The excitation map represents the tip positioning with respect to the SND molecule prior to its excitation with electrons on a grid of $25 \times 25 \mathrm{pixel}^{2}$. The molecule excitation is induced by acquiring a single current-voltage $I(V)$ spectrum above the molecule from $0 \mathrm{~V}$ to the desired voltages. For the repulsive and attractive modes, this maximum voltage applied to the tip was set to $\pm 1.8 \mathrm{~V}$, respectively. Successful translations are observed in the curve as an abrupt jump as described in the manuscript Figure 5. A total of more than 200 manipulations are reported in the excitation map of Figure 7 corresponding 
to a cumulated distance of $\sim 120 \mathrm{~nm}$. Note also that the excitation map strongly depends on the tip apex conditions. Both the indentation and manipulation procedures were successfully transferred to the four-probe microscope hosting the NanoCar Race.

Force Spectroscopic Measurements. Force spectroscopic measurements were obtained with oscillation amplitude of $A=50 \mathrm{pm}$ at $4.7 \mathrm{~K}$ without applying tip-sample voltages $(V$ $=0 \mathrm{~V})$. To built-up the $\Delta f(x, z)$ fields of Figure 8, successive frequency shift versus $x$ distance curves, $\Delta f(x)$ (grey curves) acquired from left to right above molecules at elbow and valley sites while reducing the tip-sample distance by $300 \mathrm{pm}$ with steps of $30 \mathrm{pm}$. From the $\Delta f(x, z)$ data, we extracted the potential $U(x, z)$ and lateral force fields $F_{L}(x, z)$ through successive integrations along $z$ of the $\Delta f$ and a derivation along $x$ as described in references. ${ }^{11,40-42}$ The long-range van der Waals background was estimated and extracted from the raw data using a $10-\mathrm{nm}$ long $\Delta f(z)$ curve above the gold surface.

DFT Calculations. Periodic DFT calculations were performed using the Vienna ab-initio Simulation Package (VASP) version 5.4.1. ${ }^{44}$ The simulations used projected augmented wave $(\mathrm{PAW})^{45}$ in the generalized gradient approximation (GGA) ${ }^{46}$ of the density functional. Prior to the simulations, a $7 \times 7$ super cell of the gold surface containing 6 layers was fully relaxed. During this relaxation, the bottom two layers were fixed at their bulk positions and the system was relaxed until the maximum force was of $10^{-3} \mathrm{eV} / \AA$. A vertical separation of 2.5 $\mathrm{nm}$ was introduced between the stacked unit cells. Due to the large size of the system only one $k$-point (the $\Gamma$-point) was considered during the simulations. The position of the molecule on the surface was then optimized using different starting positions. The presence of a large number of local minimas in potential energy surface of absorption of the molecule on the surface, each characterized by a specific orientation of the molecule, made the determination of a global minimum difficult. The configuration shown on Figure 3a was the most stable of all the configurations obtained. The difference density $\Delta \rho=\rho_{\text {tot }}-\left(\rho_{\text {surf }}+\rho_{\text {mol }}\right)$ was computed to visualize the modification of the electronic structure induced by the absorption 
of the molecule. A Bader population analysis was then performed to estimate the amount of charge transferred between the surface and the molecule.

Energy Barrier Diffusion. The Nudged Elastic Band (NEB) approach was first used to determine the energy barrier for diffusion. These calculations showed that the molecular conformation does not significantly change along these path, allowing to study diffusion by simple rigid displacement of the molecule on the surface. Due to the complexity of the potential energy surface for diffusion, different path were considered to better understand the diffusion process. Calculations using van-der-Waals corrected functionals based on the Tkatchenko-Scheffler approach were performed on the conformations corresponding to the local energy minimum and maximum to estimate the diffusion barrier.

AFM Image Simulation. The simulations of the AFM pictures were done using the Probe Particle Model software developed by P. Hapala et al. ${ }^{39}$ During the simulations, a probe stiffness of $0.5 \mathrm{~N} / \mathrm{m}$ was considered with a charge of -0.05 and oscillation amplitude of $3 \AA$. The full Hartree potential of the system generated by VASP was used to simulate the electrostatic interactions between the tip and the atoms in the system.

Electron-Phonon Coupling Calculation. The calculations of the vibration modes of the molecule and their interaction with the charge carriers were computed using the Amsterdam Density Functional (ADF2016) package. ${ }^{47}$ These simulations relied on a DIM-QM approach $^{48}$ where only the molecule was treated at the DFT level of theory and its interactions with the surface were described by a Polarizability Interaction Model (PIM). In this frame, a large gold surface containing 2 layers each composed of 100 atoms was considered. The vibration modes of the molecule were calculated by diagonalization of the dynamical matrix. The interaction between the charge carriers and the vibration modes were calculated following 49 


$$
V_{q}=\frac{1}{2 M_{q} \omega_{q}^{2}}\left(\frac{\partial \epsilon_{m}}{\partial R_{q}}\right)_{R=0}^{2}
$$

where $M_{q}$ is the effective of the $q$-th mode with angular frequency $\omega_{q}$ and normal-mode coordinate $R_{q} . \epsilon_{m}$ is the energy of the $m$-th orbital of the molecule. The energies of the HOMO and LUMO were here considered to compute the coupling strength with holes and electrons respectively. The derivative was numerically evaluated with a 2 points and 4 point central difference approximation to check for numerical convergence.

\section{Associated Contents}

\section{Supplementary Information}

The Supporting Information is available free of charge on the ACS Publications website at DOI: !!!

Additional STM image of the gold surface; DFT calculation of another absorption site of the flat "valley" conformation; DFT calculation of the molecular orbitals

\section{Author Informations}

\section{Corresponding Authors}

*E-mails: remy.pawlak@unibas.ch; ernst.meyer@unibas.ch

\section{ORCID}

Rémy Pawlak : 0000-0001-8295-7241

\section{Notes}

The authors declare no competing financial interest.

\section{Author Contributions:}


R.P., C.H. and E.M. conceived the experiments. R.P. performed the STM/AFM measurements at the University of Basel. N.R. performed the DFT calculations. T.M and R.P. performed the STM experiments at the CEMES laboratory with the help of D.S., C.D., S.W.H and C.J. R.P. analysed the data and wrote the manuscript. All authors discussed on the results and revised the manuscript.

\section{Acknowledgements}

This work was supported by the Swiss National Science Foundation (SNF), the Swiss Nanoscience Institute (SNI) and the COST-action MP1303. Dr. Jonas Schoenle is gratefully acknowledged for the molecule synthesis. The Swiss NanoCar Team acknowledges their sponsors the SNI, Universitäts-Kinderspital beider Basel (UKBB) and Bihr.

\section{References}

(1) Feynman, R. "Infinitesimal Machinery", presented at Jet Propulsion Laboratory on February 23, 1983; reprinted in J. Micromechanical Systems 1993, 2, 4-14.

(2) Joachim, C.L; Rapenne, G. Molecule Concept Nanocars: Chassis, Wheels, And Motors? ACS Nano 2013, 7, 11-14.

(3) Gross, L., Mohn, F., Moll, N., Liljeroth, P.; Meyer, G. The Chemical Structure of a Molecule Resolved by Atomic Force Microscopy. Science 2009, 325, 1110-1114.

(4) Eigler, D. M.; Schweizer, E. K. Positioning Single Atoms With a Scanning Tunneling Microscope. Nature 1990, 344, 524-526.

(5) Hla, S. W., Braun, K. F. \& Rieder, K. H. Single-Atom Manipulation Mechanisms During a Quantum Corral Construction. Phys. Rev. B 2003, 67, 201402 
(6) Jung, T. A., Schlittler, R. R., Gimzewski, J. K., Tang, H.; Joachim, C. Controlled Room-Temperature Positioning of Individual Molecules: Molecular Flexure And Motion. Science 1996, 271, 181.

(7) Moresco, F.; Meyer, G.; Reider, K.-H.; Tang, H.; Gourdon, A.; Joachim, C. Recording Intramolecular Mechanics During The Manipulation of a Large Molecule. Phys. Rev. Lett. 2001, 87, 088302.

(8) Grill, L., Rieder, K. H.; Moresco, F. Exploring The Interactomic Forces Between a Tip And Single Molecules During STM Manipulation. Nano Lett. 2006, 12, 2685-2689.

(9) Pawlak, R.; Fremy, S.; Kawai, S.; Glatzel, T.; Fang, H.; Fendt, L.-A.; Diederich, F. Meyer, E. Directed Rotations of Single Porphyrin Molecules Controlled by Localized Force Spectroscopy. ACS Nano 2012, 6, 6318-6324.

(10) Kawai, S.; Koch, M.; Gnecco, E.; Sadeghi, A.; Pawlak, R.; Glatzel, T.; Schwarz, J.; Goedecker, S.; Hecht, S.; Baratoff, A.; Grill, L.; Meyer, E. Quantifying The AtomicLevel Mechanics of Single Long Physisorbed Molecular Chains. Proc. Natl. Acad. Sci. USA 2014, 111, 3968-3972.

(11) Kawai, S.; Benassi, A.; Gnecco, E.; Söde, H.; Pawlak, R.; Feng, X.; Müllen, K.; Passerone, D.; Pignedoli, C.A.; Ruffieux, P.; Fasel, R.; Meyer, E. Superlubricity of Graphene Nanoribbons on Gold Surfaces Science 2016, 351, 957-961.

(12) Pawlak, R.; Ouyang, W.; Filippov, A.E.; Kalikhman-Razvozov, L.; Kawai, S.; Glatzel, T.; Gnecco, E.; Baratoff, A.; Zheng, Q.S.; Hod, O.; Urbakh, M.; Meyer, E. SingleMolecule Tribology: Force Microscopy Manipulation of a Porphyrin Derivative on a Copper Surface. ACS Nano 2016, 10, 713-722.

(13) Pawlak, R.; Kawai, S.; Meier, T.; Glatzel, T.; Baratoff, A.; Meyer, E. Single-Molecule Manipulation Experiments to Explore Friction And Adhesion. J. Phys. D: Appl. Phys. 2017, 50, 113003. 
(14) Shirai, Y., Osgood, A. J., Zhao, Y., Kelly, K. F.; Tour, J. M. Directional Control in Thermally Driven Single-Molecule Nanocars Nano Lett. 2005, 5, 2330-2334.

(15) Kudernac T.; Ruangsupapichat, N.; Parschau, M.; Maciá, B.; Katsonis, N.; Harutyunyan, S. R.; Ernst, K.-H.; Feringa, B. L. Electrically Driven Directional Motion of a Four-Wheeled Molecule on a Metal Surface. Nature 2011, 479, 208-211.

(16) Pascual, J. I.; Lorente, N.; Song, Z.; Conrad, H.; Rust, H.-P. Selectivity in Vibrationally Mediated Single-Molecule Chemistry. Nature 2003, 423, 525-528.

(17) Swart, I.; Sonnleitner, T.; Niedenführ, J.; Repp, J. Controlled Lateral Manipulation of Molecules on Insulating Films by STM. Nano Lett. 2012, 12, 1070-1074.

(18) Sonnleitner, T.; Swart, I.; Pavliček, N.; Pöllmann A.; Repp J. Molecular Symmetry Governs Surface Diffusion. Phys. Rev. Lett. 2011, 107, 186103.

(19) Stipe, B. C.; Rezaei, M. A.; Ho, M. A. Inducing And Viewing The Rotational Motion of a Single Molecule. Science 1998, 279, 1907-1909.

(20) Stipe, B. C.; Rezaei, M. A.; Ho, W. Coupling of Vibrational Excitation to The Rotational Motion of a Single Adsorbed Molecule. Phys. Rev. Lett. 1998, 81, 1263.

(21) Komeda, T.; Kim, Y.; Kawai, M.; Persson, B. N. J.; Ueba, H. Lateral Hopping of Molecules Induced by Excitation of Internal Vibration Mode. Science 2002, 295, 20552058.

(22) Hla, S.-W.; Bartels, L.; Meyer, G.; Rieder, K.-H. Inducing All Steps of a Chemical Reaction With The Scanning Tunneling Microscope Tip: Towards Single Molecule Engineering. Phys. Rev. Lett. 2000, 85, 2777.

(23) Harikumar, K. R.; Polanyi, J.C.; Zabet-Khosousi, A.; Czekala, P.; Lin, H.; Hofer, W.A. Directed Long-Range Molecular Migration Energized by Surface Reaction. Nature Chemistry 2011, 3, 400-408. 
(24) Pavliček, N.; Schuler, B.; Collazos, S.; Moll, N.; Pérez, D.; Guitián, E.; Meyer, G.; Peña, D.; Gross, L. On-Surface Generation And Imaging of Arynes by Atomic Force Microscopy. Nature Chem. 2015, 7, 623-628.

(25) Schuler, B.; Fatayer, S.; Mohn, F.; Moll, N.; Pavliček, N.; Meyer, G.; Penña, D.; Gross, L. Reversible Bergman Cyclization by Atomic Manipulation. Nature Chem. 2016, 8, $220-224$.

(26) Echeverria, J., Monturet, S.; Joachim, C. One-Way Rotation of a Molecule-Rotor Driven by a Shot Noise. Nanoscale 2014, 6, 2793-2799.

(27) Ohara, M.; Kim, Y.; Kawai, M. Electric Field Response of a Vibrationally Excited Molecule in an STM Junction. Phys. Rev. B 2008, 78, 201405.

(28) Repp, J.; Steurer, I. S.; Persson, M.; Gross, L.; Meyer, G. Charge-State-Dependent Diffusion of Individual Gold Adatoms on Ionic Thin NaCl Films. Phys. Rev. Lett 2016, $117,146102$.

(29) Nickel, A.; Ohmann, R.; Meyer, J.; Grisolia, M.; Joachim, C.; Moresco, F.; Cuniberti, G. Moving Nanostructures: Pulse-Induced Positioning of Supramolecular Assemblies. ACS Nano 2013, 9, 8394-8400.

(30) Ohmann, R.; Meyer, J.; Nickel, A.; Echeverria, J.; Grisolia, M.; Joachim, C.; Moresco, F.; Cuniberti, G. Supramolecular Rotor And Translator at Work: On-Surface Movement of Single Atoms. ACS Nano 2015, 7, 191-197.

(31) Eisenhut, F.; Durand, C.; Moresco, F.; Launay, J.-P.; Joachim, C. Training For The $1^{s t}$ International Nano-Car Race: The Dresden Molecule-Vehicle. Eur. Phys. J. Appl. Phys. 2016, 76, 10001.

(32) Constable, E. C.; Housecroft, C; Price, J. R.; Zampese, J. A. When Five Are Six: The 
Myth of Five-Coordinate II) in Supramolecular Chemistry. CrystEngComm 2010 12, $3163-3171$.

(33) Grillo, F.; Früchtl, H.; Francis, S. M.; Richardson, N. V. Site Selectivity in The Growth of Copper Islands on $\mathrm{Au}(111)$. New J. Phys. 2011, 13, 01344.

(34) Cheng, Z. H.; Gao, L.; Deng, Z.T.; Jiang, N.; Liu, Q.; Shi, D.X.; Du, S.X.; Guo, H.M.; Gao H.-J. Adsorption Behaviour of Iron Phthalocyanine on Au(111) Surface at Submonolayer Coverage. J. Phys. Chem. C 2007, 111, 9240-9244.

(35) Wang, Y.; Hush, N. S.; Reimers, J. R. Simulation of The Au(111)- $(22 \times \sqrt{3})$ Surface Reconstruction. Phys. Rev. B 2007, 75, 233416.

(36) Hanke, F.; Björk, J. Structure And Local Reactivity of The Au(111) Surface Reconstruction. Phys. Rev. B 2013, 87, 235422.

(37) Kocić, N.; Liu, X.; Chen, S.; Decurtins, S.; Krejčí, S.; Jelínek, P.; Repp, J.; Liu, S.-X. Control of Reactivity and Regioselectivity for On-Surface Dehydrogenative Aryl-Aryl Bond Formation. J. Am. Chem. Soc. 2016, 138, 5585-5593.

(38) Zint, S.; Ebeling, D.; Schlöder, D.; Ahles, S.; Mollenhauer, D.; Wegner, H. A.; Schirmeisen, A. Imaging Successive Intermediate States of the On-Surface Ullmann Reaction on $\mathrm{Cu}(111)$ : Role of the Metal Coordination. ACS Nano 2017, 11, 41834190.

(39) Hapala, P.; Kichin, G.; Wagner, C.; Tautz, F. S.; Temirov, R.; Jelìnek, P. Mechanism of High-Resolution STM/AFM Imaging With Functionalized Tips. Phys. Rev. B 2014, 90, 085421 .

(40) Ternes, M.; Lutz, C. P.; Hirjibehedin, C. F.; Giessibl, F. J.; Heinrich, A. J. The Force Needed to Move an Atom on a Surface. Science 2008, 319, 1066-1069. 
(41) Langewisch, G.; Falter, J.; Fuchs, H.; Schirmeisen, A. Forces During The Controlled Displacement of Organic Molecules. Phys. Rev. Lett. 2013, 10, 036101.

(42) Jarvis, S. P.; Taylor S.; Baran, J. D.; Champness, N. R.; Larsson, J. R.; Moriarty, P. Measuring The Mechanical Properties of Molecular Conformers. Nature Comm. 2014, 6,8388 .

(43) Meyer, J.; Nickel, A.; Ohmann, R.; Lokamani; Toher, C.; Ryndyk, D. A.; Garmshausen, Y.; Hecht, S.; Moresco, F.; Cuniberti, G. Tuning The Formation of Discrete Coordination Nanostructures. Chem. Comm. 2015, 51, 12621.

(44) Kresse, G.; Hafner, J. Ab-initio Molecular Dynamics For Liquid Metals, Phys. Rev. B 1993, 47, 558 .

(45) Kreese, G.; Joubert, D. From Ultrasoft Pseudopotentials to The Projector AugmentedWave Method. Phys. Rev. B 1999, 59, 1758.

(46) Perdew, J. P.; Burke, K.; Ernzerhof, M. Generalized Gradient Approximation Made Simple. Phys. Rev. Lett. 1996, r7, 3865.

(47) te Velde, G.; Bickelhaupt, F.M.; van Gisbergen, S.J.A.; Fonseca Guerra, C.; Baerends, E.J.; J.G. Snijders; Ziegler, T. Chemistry With ADF. J. Comp. Chem. 2001, 22, 931.

(48) Payton, J. L.; Morton, S. M.; Moore, J. E.; Jensen, L. A Discrete Interaction Model/Quantum Mechanical Method For Simulating Surface-Enhanced Raman Spectroscopy. J. Chem. Phys. 2012, 136, 214103.

(49) Coropceanu, V.; Cornil, J.; da Silva Filho, D.A.; Olivier, Y.; Silbey, R.; Brédas, J.-L. Charge Transport in Organic Semiconductors. Chem. Rev. 2007, 107, 926-952. 
Graphical TOC Entry

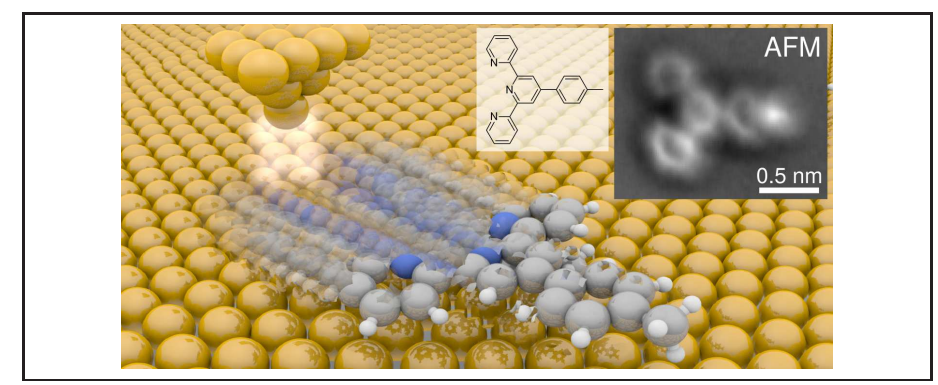




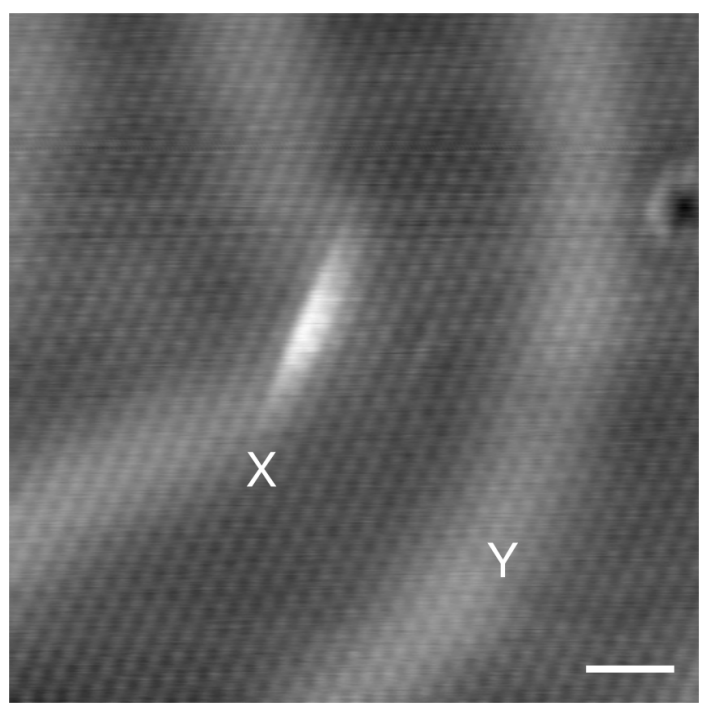

Figure S1: The Gold Surface. STM image of the Au(111) surface with atomic resolution showing the $X$ and $Y$ elbows. The structural peculiarities of the $X$ elbow leads to the complex formation with the SND described in the main manuscript.
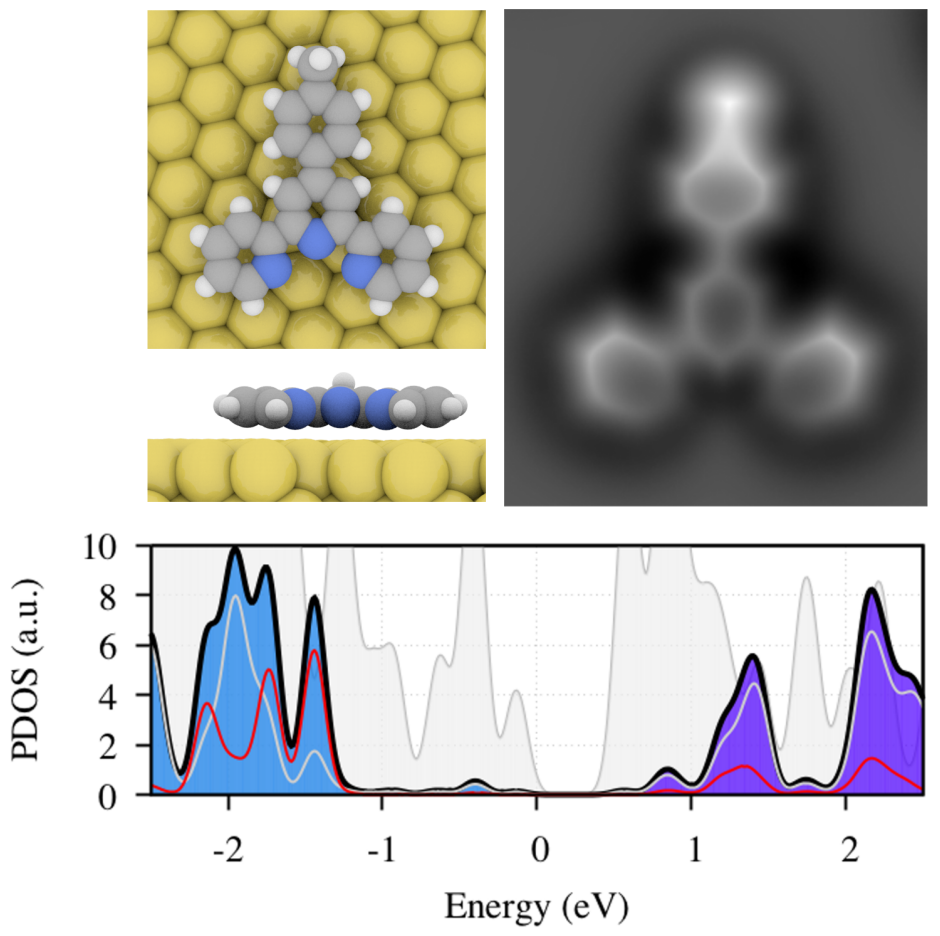

Figure S2: Another Absorption Site of The Flat "Valley" Conformation. a, Top and side views of a metastable absorption geometry of the flat conformation obtained by DFT optimization using VASP. b, Simulated AFM image almost identical to the one represented in Figure 2. c, Projected density of states of the system showing the contribution of the gold (grey shaded area), carbon (grey line) and nitrogen (blue line). The total PDOS of the molecule is shown as blue and purple shaded area for the occupied and unoccupied states. 
As mentioned in the main text the absorption of the molecule on the surface present a large number local minimas. One of the metastable conformation is represented in Fig. S2. At the difference of the conformation shown in Figure 2 of the main text, the molecule is here not aligned with the $\mathrm{Au}-\mathrm{Au}$ bonds. This allows for each benzene ring to sit on top of a gold atom hence increasing the $\pi$-stacking interaction between the molecule and the surface. However in such conformation the lone pairs of the nitrogen atoms are not coordinated with the gold atoms, which overall decrease the molecule-surface interactions. This different absorption conformation illustrate the complexity of the absorption energy landscape. Therefore while the conformation shown in Fig. 2 is the most stable we have found a more stable one might still exists. 


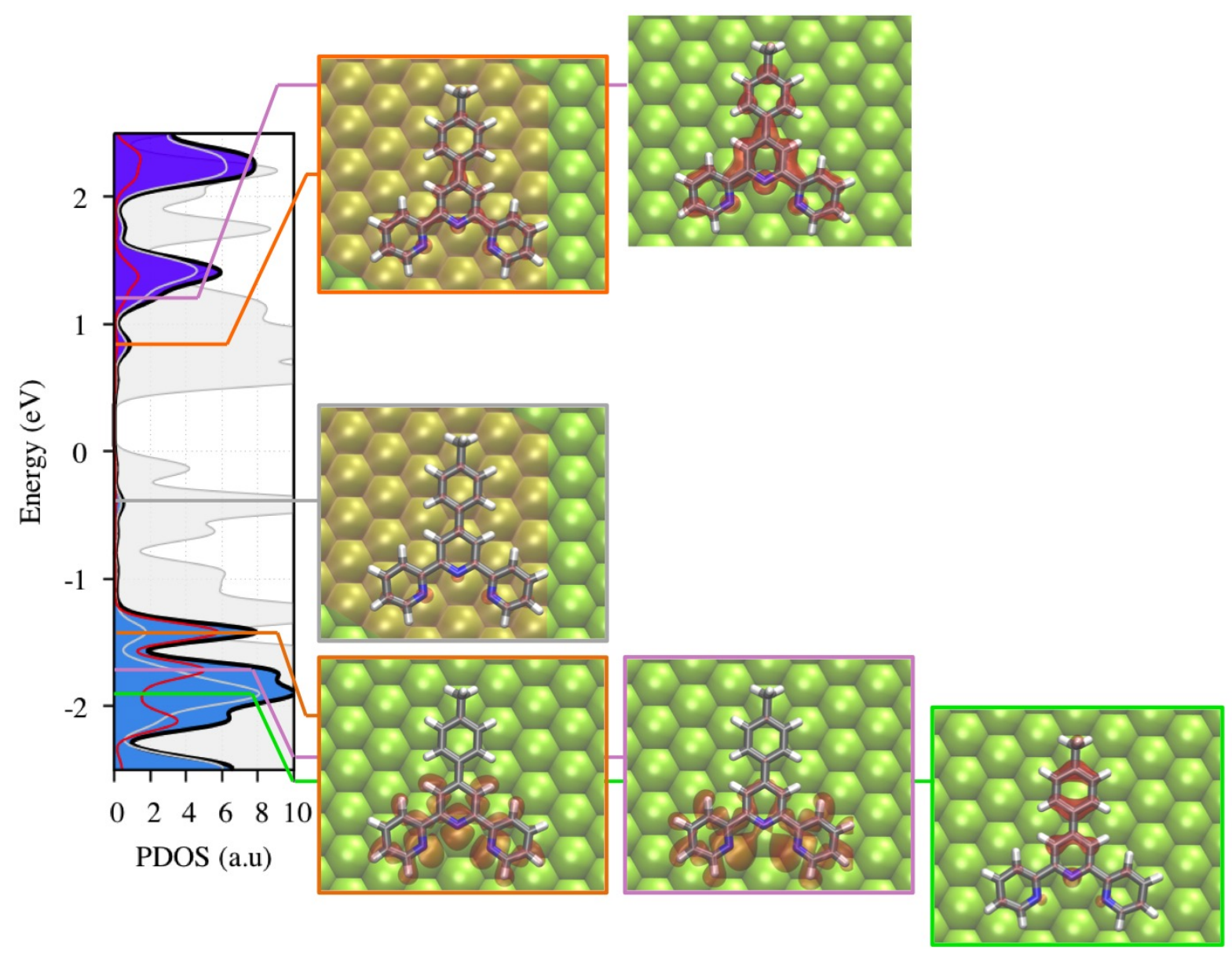

Figure S3: Molecular Orbitals obtained via integration of the total density of states between selected energy values.

Molecular orbitals were obtained by integrating the total density of states between selected energy values delimiting the features observed on the PDOS. These orbitals are shown on Fig. S3. As seen on this figure, the small features at $\sim-0.5 \mathrm{eV}$ and $\sim 0.8 \mathrm{eV}$ are mainly due to gold states with only a small contributions from the molecular states. However the large peaks $\sim-1.5 \mathrm{eV}$ correspond to the $\sigma$ network of the pyridine rings with a strong contribution of the nitrogen lone pairs. The peak at $-1.8 \mathrm{eV}$ corresponds to a $\pi$ orbital of the benzene ring with a small contribution of the nitrogen lone pairs. The LUMO of the molecule present an energy of about $1.1 \mathrm{eV}$ and is delocalized principally over the $p i_{z}$ atomic orbitals of the carbon atoms. 